\title{
Fundamentals of Optical Interconnections-a Review
}

\author{
Haldun M. Ozaktas \\ Bilkent University, Department of Electrical Engineering \\ TR-06533 Bilkent, Ankara, Turkey
}

\begin{abstract}
We review some of the relatively fundamental work in the area of optically interconnected digital computing systems. We cover comparisons of optical interconnections with other interconnection media in terms of energy and interconnection density, studies determining the optimal combination of optical and electrical interconnections that should be used, work on free-space optical interconnection architectures, complexity studies, and work on physical and logical system architectures and algorithms. We exclude work on devices, components, materials, and manufacturing.
\end{abstract}

\section{Introduction}

Following publications such as [1], a substantial amount of effort has been invested to determine whether, when, and how the use of optical interconnections may lead to improvements in digital computing systems. These efforts have been multi-faceted, ranging from the development of materials, devices, and components; to system-level studies comparing the relative strengths and weaknesses of optical interconnections; to the development of novel optical and system architectures.

In this work we attempt to provide a review of some of the relatively fundamental work in this area. We exclude fundamental work on devices, materials, and manufacturing techniques. We also limit our attention to digital systems, excluding analog systems and neural networks. We further exclude other important topics such as computer-aided design of optoelectronic or optically interconnected systems.

We cannot claim to be comprehensive, neither in terms of content nor in terms of the authors cited. Space limitations has allowed us to include only a sampling of references. We will be grateful for omissions brought to our attention, as well as suggestions regarding the organization of this paper. An attempt will be made to incorporate these in the full version of this paper.

\section{General considerations}

The following considerations are not specific to optically interconnected systems but apply to all digital computers.

\subsection{Heat removal limitations}

The nonlinear elements (switches or gates) of which digital computing systems are universally made of, dissipate a certain amount of energy $E_{d}$ per switching event. Furthermore, each bit of information that is sent from one point to another is represented by an amount of energy $E$, which is consequently dissipated. The value of $E_{d}$ may very well be in the $\mathrm{fJ}$ range. On the other hand, the value of $E$ will more likely be of the order of a $\mathrm{pJ}$ or more. Smaller values may be attainable for very short interconnections, but for systems whose overall size is of the order of $1 \mathrm{~m}$, it seems fair to take $E+E_{d} \approx E \sim 1 \mathrm{pJ}$ as an appropriate average for the energy per switching/transmission event.

If we let $N$ denote the number of elements (switches or gates) in our system and $k$ the average number of interconnections per switch, then the total power dissipated is $\mathcal{P}=k N E B$, where $B$ is the bit-repetition-rate; the rate at which bits of information are being emitted into each interconnection. With $N=10^{8}, k=5, B=2 \mathrm{~Gb} / \mathrm{s}$, we obtain $\mathcal{P}=1 \mathrm{MW}$. If this system occupies a volume of $\mathcal{L}^{3}=(10 \mathrm{~cm})^{3}$, then it becomes necessary to remove $10^{8} \mathrm{~W} / \mathrm{m}^{2}=10 \mathrm{~kW} / \mathrm{cm}^{2}$ through the system. This seems to be the very upper limit of what can be achieved by fluid convection [2], and would probably be very difficult to achieve. Notice that the amount of power we can remove from a system is proportional to the cross-sectional area (not the surface area) of the system [2].

Simple calculations will show that the space occupied by the elements and even the interconnections is small in comparison. The moral is that heat removal will be the most important consideration in high performance future computing systems. It will be of paramount importance to employ the most aggressive heat removal methods and integrate heat removal into system design from the onset [3]. 


\subsection{Confining active devices to planes}

For highly interconnected circuits, the following result is of relevance $[4,5,6]$ : Provided the interconnections are allowed to be routed through three-dimensional space, there is no disadvantage in restricting the active devices to a plane. That is, the overall volume and signal delay and thus clock rate of a highly interconnected three-dimensional system in which the active devices are restricted to a plane will not be inferior to a system in which the active devices can be situated freely throughout the volume. This is because the volume is primarily determined by heat removal limitations or the space occupied by the interconnections, so that additional restrictions on the active devices are of little or no significance. This result, which is based on purely geometrical considerations, is valid for all types of interconnections.

\subsection{Globally interconnected systems}

Globally interconnected systems are better than locally interconnected systems because global connections allow flexible and fast global transfer of information. Global interconnections take up much more space, but since heat removal considerations imply large separations between the elements anyway, this does not result in larger system size and delays [7].

\section{Energy comparisons}

Having concluded that heat removal limitations will become one of the most important, if not the most important consideration, it follows that reducing the energy per transmitted bit of information $E$ is of primary importance. Various studies comparing $E$ for isolated optical and electrical lines indicate that optics becomes more profitable for interconnections shorter than some break-even distance, estimated between $0.1 \mathrm{~mm}$ and $10 \mathrm{~cm}[8,9,10]$. (These comparisons assume that the energy received is large enough to charge the receiver capacitance to the nominal logic levelarea consuming amplifier circuits as used in long-distance communications would be unacceptable.)

Studies which account for a very large number of parameters exist, but the essence of the comparison can be captured by a simple argument. Since attenuation in optical media is very low (as evident from the distance information is able to travel in telecommunications fibers), the energy per transmitted bit can be assumed to be constant in optical systems. On the other hand, the energy for an unterminated (lumped RC) conducting interconnection is proportional to $\ell^{2}$, where $\ell$ is the length of the line. Thus, beyond a certain value of $\ell$, optical interconnections are deemed favorable. If terminated lines are used, the energy is proportional to the length $T$ of the shortest pulse that can propagate on the line with acceptable attenuation, which is in turn proportional to $\ell^{2} / W^{2}$, where $W$ is the width of the line. The only way to prevent the energy from increasing with length is to increase $W \propto \ell$, but this increase in the thickness of lines and subsequent crowding will eventually result in an increase in the size of the system, increasing the line lengths $\ell$, which will require a further increase in $W$, and so on, thwarting the attempt to circumvent the intrinsic limitations associated with line resistance.

Miller has given an intriguing physical interpretation of the fact that optical interconnections are energetically more advantageous [10]. Other fundamental works in this area include $[11,12,13]$. The effect of fan-in and fan-out is discussed in [14].

It has been shown that the energy per transmitted bit with superconducting interconnections is also constant and comparable to what may be achieved with optical interconnections, for a nominal voltage level of $1 \mathrm{~V}$. It may be further reduced if lower nominal voltages are employed at lower temperatures [4].

\section{Interconnection density}

\subsection{Comparisons with other technologies}

A fairly common misconception is that optical channels do not occupy any volume and are "free" in this sense. Whereas it is true that separate optical channels can cross through each other with little or no interference, optical channels nevertheless have a volume cost: The minimum volume occupied by an optical interconnection system with total interconnection length $\ell_{\text {total }}$ is $\sim \lambda^{2} \ell_{\text {total }}$, where $\lambda$ is the wavelength of light [15]. Thus, each independent spatial channel can be thought to have a constant transverse area of $\lambda^{2}$.

On the other hand, the cross-sectional area of conducting interconnections must increase with length to maintain acceptable attenuation. Thus beyond a certain length, optical interconnections become favorable. The break-even lengths cited are comparable to those obtained from energy considerations $[4,8,16,17]$.

Two-dimensional integrated optical guided wave circuits are not able to support a surface density of one spatial channel per wavelength because of the larger separations implied by crosstalk considerations. Such systems will probably not be favorable until extremely large system sizes $[18,19]$.

\subsection{Topological considerations for optics}

The word "minimum" appearing above is of significance. Indeed, it was first thought that a strong tradeoff between topological flexibility (the ability to implement arbitrary patterns of connections) and volume existed: that greater 
flexibility came at the cost of greater volume. However, it was later understood that these tradeoffs applied only to a broad class of architectures which may be referred to as multi-facet architectures, but that other architectures free from these limitations are possible. Thus arbitrary circuit diagrams (graphs) can be realized with an effective interconnection density of $\sim 1 / \lambda^{2}$. This result assumes optical systems whose $f$-numbers (analogous to the $F$-stop on a camera) approach unity. In practice, it seems realistic to expect this density to be achieved within an order of magnitude. In most cases this will be sufficient, since heat removal and not interconnection density will be the limiting factor.

Publications investigating these issues include $[20,21$, $22,23,24,25,26,27,28,29]$

\subsection{Architectures for free-space optics}

A large variety of free-space optical interconnection architectures have been proposed and studied. In this section we mention works that deal with the optical interconnections only, without consideration of the overall computing system architecture $[30,31,32,33,34,35,36,37,38,39,40,41]$. These studies concentrate primarily on optical systems to image arrays of sources onto arrays of detectors in an efficient manner. Several authors have discussed the ultimate limitations of these systems; others have been led to investigations of mathematical and combinatoric issues regarding permutation and other types of networks. Shuffle-based systems are by far the most commonly studied both because they have efficient optical implementations and because of their versatility.

"Planar free-space" systems also deserve mention in this context [42].

\section{System-level studies}

\subsection{Area-volume-time complexity studies}

Here we are referring to area-volume-time studies either in the spirit of classical VLSI complexity theory [43], or the closely related pin-out/line-length distribution based studies $[44,45]$. Work on three-dimensional complexity theory includes $[4,5,6,46,47,48]$. Some of these works are classical complexity studies, being based on purely geometrical considerations and ignoring all constants of proportionality. Others have reintroduced physical constants and order-ofmagnitude accuracy in an attempt to obtain results which are more physically relevant [4].

\subsection{System-level technology comparisons}

These studies typically assume an array of elements of some degree of complexity (usually ranging from transistors to processors) and compare performance and cost measures resulting from the use of different interconnection media. These works differ from the comparison studies mentioned earlier in that they compare systems, rather than isolated interconnections $[4,49,50,51]$.

Based solely on fundamental physical considerations, optical and superconducting interconnections are comparable to each other and superior to normally conducting interconnections, even when aggressive use of repeaters is made to circumvent capacitive and resistive problems. The possibility of three-dimensional circuits and freedom from termination problems seems to give optics an edge over superconductors.

The use of normally conducting interconnections results in severe limitations in large scale systems. Even with arbitrarily small scaling and arbitrarily fast devices, with or without termination, the maximum bit rates that can be supported sharply decrease with increasing system size. Likewise, the bisection-inverse delay and bisection-bandwidth products are bounded from above. This is in contrast with the other media with which it is possible to arbitrarily increase $B$ and the bisection-bandwidth product for any given $N$. The use of repeaters alleviates, but does not change the inferiority of normal conductors, while at the same time greatly increasing manufacturing complexity because of the need to support active devices at all levels of the interconnection hierarchy. The use of optics is comparably even more advantageous when large bandwidths are required.

\subsection{Optimal combination of optical and electrical interconnections}

These studies discuss how optical and electrical interconnections can be used together. They essentially address the question: Beyond what level of the interconnection hierarchy should optics be used? Or, how should a large circuit be partitioned into optically interconnected electronic chips? That is, what is the optimal grain size? $[19,52,53,54]$

The general indication is that the system should be partitioned into clusters of $\sim 10^{4}-10^{6}$ transistors; for instance, in the form of an optically interconnected array of chips with this many transistors on each. It has been argued that the use of optical interconnections allows one to access points in performance space unachievable otherwise, however possibly at a significant cost of space and/or power [19]. The increase in performance may take the form of smaller signal delay and/or larger bandwidth in comparison to an all-electrical system. If minimization of the cost associated with size and power is the primary objective, rather than the maximization of performance, then the use of optics does not become beneficial until extremely large system sizes. 


\subsection{Multi-faceted system-level studies}

Here we mention some studies which deal with many or all aspects of an optically interconnected system simultaneously, rather than concentrating on only one part (such as the smart pixel array or the optical interconnection architecture). Some of these studies are part of a specific development effort, but the results nevertheless have general applicability or implications. $[55,56,57,58,59]$

Figure 1 depicts the tree of alternative optical interconnection architectures. In [60] we started from the top of this tree and by offering arguments that allow us to prune suboptimal branches, we finally arrived at the boxed alternative shown at the bottom as the basis for an optimal architecture.

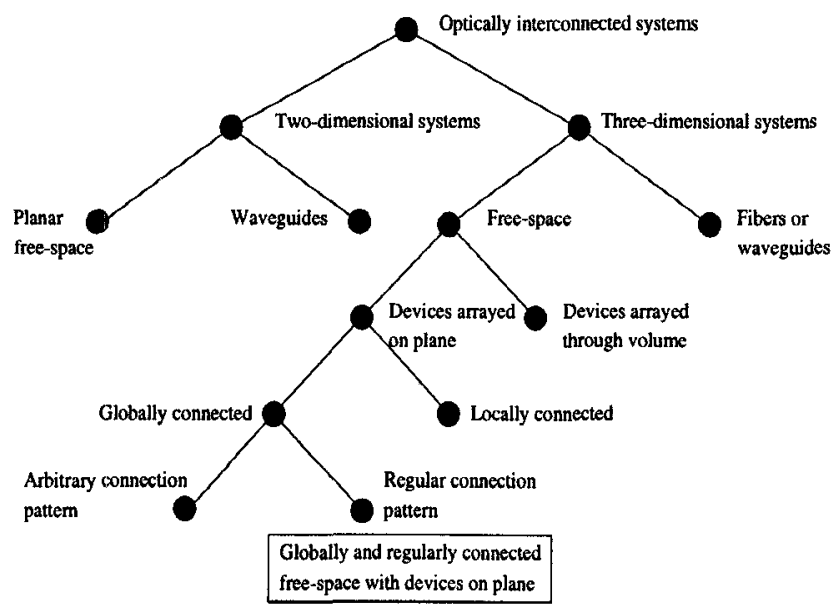

Figure 1.

\subsection{Physical system architecture and technology platform development}

Many different systems employing optical interconnections in one form or another have been or are being developed. Although these specific development efforts may not be considered fundamental, they are important because they represent novelties or breakthroughs in design, architecture, packaging, or systems engineering $[61,62,63,64,65,66,67,68,69,70]$.

It should be noted that considerable subjectivity is involved in determining what should be included in this or the preceding subsection.

\subsection{Algorithms and logical architectures}

The different physical architectures supported by optics has created interest in different logical architectures and associated algorithms which better match the abilities of optical interconnections. These often involve massive parallel flows of information [71]. In addition to exploration of various different paradigms (systolic architectures, connectionist systems, cellular systems, etc.), there has also been a revival of work in switching networks $[62,72,73,74]$.

\subsection{Components and devices}

While we have excluded components and devices from the scope of this paper, a number of studies deserve inclusion because of their fundamental implications from a systems viewpoint. For instance, we may mention device comparison studies such as $[75,76]$, and studies analyzing the scaling properties of refractive and diffractive lenses $[77,78]$.

Other publications deal with the design of optical components specifically for optical interconnection applications. These include the design of diffractive optical components [79], microoptical assemblies, and spot array generators.

\section{Technology evolution studies}

These studies may help track the evolution of optoelectronic technology in the context of the history of development of digital computing systems in general, and may also shed light on why a technology with fundamental credentials as strong as optics, is nevertheless not easily able to find its way into mainstream systems $[80,81,82]$.

\section{Conclusion}

Although a consolidated body of knowledge and understanding has emerged in several areas, this is particularly not the case for full-blown system-level studies. The different starting points and assumptions makes it difficult to compare the many studies on a common footing. This may be viewed as a strength as much as a weakness, as the community has not been locked into a specific direction which may be suboptimal.

\section{References}

[1] J. W. Goodman, F. J. Leonberger, S.-Y. Kung, and R. Athale. Optical interconnections for VLSI systems. Proc IEEE, 72:850-866, 1984.

[2] H. M. Ozaktas, H. Oksuzoglu, R. F. W. Pease, and J. W. Goodman. Effect on scaling of heat removal requirements in three-dimensional systems. Int J Electronics, 73:1227-1232, 1992.

[3] W. Nakayama. Heat-transfer engineering in systems integrationoutlook for closer coupling of thermal and electrical designs of computers. IEEE Trans Components, Packaging, and Manufacturing Technology, Part A, 18:818-826, 1995.

[4] H. M. Ozaktas and J. W. Goodman. The limitations of interconnections in providing communication between an array of points. In Frontiers of Computing Systems Research, Volume 2, Plenum Press, New York, 1991, pp 61-130. 
[5] A. L. Rosenberg. Three-dimensional VLSI: a case study. $J A C M$. $30: 397-416,1983$.

[6] F. T. Leighton and A. L. Rosenberg. Three-dimensional circuit layouts. J Computer and System Sciences, 15:793-813, 1986.

[7] H. M. Ozaktas and J. W. Goodman. Comparison of local and global computation and its implications for the role of optical interconnections in future nanoelectronic systems. Opt Commun, 100:247-258, 1993.

[8] R. K. Kostuk, J. W. Goodman, and L. Hesselink. Optical imaging applied to microelectronic chip-to-chip interconnections. Appl Opt, $24: 2851-2858,1985$.

[9] M. R. Feldman, S. C. Esener, C. C. Guest, and S. H. Lee. Comparison between optical and electrical interconnects based on power and speed considerations. Appl Opt, 27:1742-1751, 1988.

[10] D. A. B. Miller. Optics for low-energy communication inside digital processors: quantum detectors, sources and modulators as efficient impedance converters. Opt Lett, 14:146-148, 1989.

[11] H. J. Caulfield and J. Shamir. Wave particle duality considerations in optical computing. Appl Opt, 28:2184-2186, 1989.

[12] H. J. Caulfield, J. Shamir, J. E. Ludman, and P. Greguss. Reversibility and energetics in optical computing. Opt Lett, 15:912-914, 1990.

[13] H. M. Ozaktas and J. W. Goodman. The optimal electromagnetic carrier frequency balancing structural and metrical information densities with respect to heat removal requirements. Opt Commun, 94:13-18, 1992.

[14] J. W. Goodman. Fan-in and fan-out with optical interconnections. Optica Acta, 32:1489-1496, 1985.

[15] H. M. Ozaktas and J. W. Goodman. Lower bound for the communication volume required for an optically interconnected array of points. J Opt Soc Am A, 7:2100-2106, 1990

[16] M. R. Feldman, C. C. Guest, T. J. Drabik, and S. C. Esener. Comparison between optical and electrical interconnects for fine grain processor arrays based on interconnect density capabilities. Appl Opt, $28: 3820-3829,1989$.

[17] D. A. B. Miller and H. M. Ozaktas. Limit to the bit rate capacity of electrical interconnects from the aspect ratio of the system architecture. I Parallel and Distributed Computing, to appear.

[18] G. Önal, A. Altıntaş, and H. M. Ozaktas. Computer-aided analysis and simulation of complex passive integrated optical circuits of arbitrary rectilinear topology. Opt Eng, 33:1596-1603, 1994

[19] H. M. Ozaktas and J. W. Goodman. Elements of a hybrid interconnection theory, Appl Opt, 33:2968-2987, 1994.

[20] J. Shamir. Fundamental speed limitations on parallel processing.Appl Opt, 26:1567, 1987

[21] R. K. Kostuk, J. W. Goodman, and L. Hesselink. Design considerations for holographic optical interconnects. Appl Opt, 26:3947-3953 1987.

[22] M. R. Feldman and C. C. Guest. Interconnect density capabilities of computer generated holograms for optical interconnection of very large scale integrated circuits. Appl Opt, 28:3134-3137, 1989.

[23] A. W. Lohmann and A. S. Marathay. Globality and speed of optical parallel processors. Appl Opt, 28:3838-3842, 1989

[24] C. W. Stirk and D. Psaltis. Comparison of optical and electronic 3-dimensional circuits. In Proc SPIE 1389, 580-593, 1990.

[25] H. M. Ozaktas, Y. Amitai, and J. W. Goodman. Comparison of system size for some optical interconnection architectures and the folded multi-facet architecture. Opt Commun, 82:225-228, 1991.

[26] A. W. Lohmann. Image formation of dilute arrays for optical information processing. Opt Commun, 86:365-370, 1991
[27] G. E. Lohman and K.-H. Brenner. Space-variance in optical computing. Optik, 89:123-134, 1992.

[28] H. M. Ozaktas and D. Mendlovic. Multi-stage optical interconnection architectures with least possible growth of system size. Opt Lett, $18: 296-298,1993$

[29] H. M. Ozaktas, K.-H. Brenner, and A. W. Lohmann. Interpretation of the space-bandwidth product as the entropy of distinct connection patterns in multifacet optical interconnection architectures. J Opt Soc Am A, 10:418-422, 1993.

[30] A. W. Lohmann. What classical optics can do for the digital optical computer. Appl Opt, 25:1543-1549, 1986.

[31] A. Lohmann, G. Stucke, and W. Stork. Optical perfect shuffle. Appl Opt, 25:1530-1531, 1986.

[32] K.-H. Brenner and A. Huang. Optical implementations of the perfect shuffle interconnection. Appl Opt, 27:135-137, 1988.

[33] C. Stirk, R. A. Athale, and M. W. Haney. Folded perfect shuffle optical processor. Appl Opt, 27:202-203, 1988.

[34] Q. W. Song and F. T. S. Yu. Generalized perfect shuffle using optical spatial filtering. Appl Opt, 27:1222-1223, 1988.

[35] J. Jahns and M. J. Murdocca. Crossover networks and their optical implementation. Appl Opt, 27:3155-3160, 1988.

[36] J. Shamir, H. J. Caulfield, and R. B. Johnson. Massive holographic interconnection networks and their limitations. Appl Opt, 28:311324,1989

[37] M. W. Haney and J. J. Levy. Optically efficient free-space folded perfect-shuffle network. Appl Opt, 30: 2833-2840, 1991.

[38] J. Giglmayr. Generation and evaluation of $d$-dimensional ( $d \geq 3$ ) optical shuffle patterns. Appl Opt, 31:4662-4675, 1992.

[39] G. C. Marsden, P. J. Marchand, P. Harvey, and S. C. Esener. Optical transpose interconnection system architectures. Opt Lett, 1083-1085, 1993.

[40] J. Giglmayr. Locality and decomposition of regular optical interconnection patterns. Appl Opt, 33:6157-6167, 1994

[41] P. S. Guilfoyle and J. M. Hessenbruch. Reconfigurable $N^{4}$ optical interconnects using the PROMAC architecture. Int J Optical Memory and Neural Networks, 3:99-109, 1994.

[42] J. Jahns. Planar packaging of free-space optical interconnections Proc IEEE, 82:1623-1631, 1994

[43] J. D. Ullman. Computational Aspects of VLSI. Computer Science Press, Rockville, Maryland, 1984.

[44] R. W. Keyes. The Physics of VLSI Systems. Addison-Wesley, Reading, Massachusetts, 1987.

[45] H. M. Ozaktas. Paradigms of connectivity for computer circuits and networks. Opt Eng, 31:1563-1567, 1992

[46] R. Barakat and J. Reif. Lower bounds on the computational efficiency of optical computing systems. Appl Opt, 26:1015-1018, 1987.

[47] M. M. Eshaghian, D. K. Panda, V. K. P. Kumar, Resource requirement for digital computations on electrooptical systems. Appl Opt, 30:928 935, 1991

[48] S. Pavel and S. G. Akl. Area-time trade-offs in arrays with optical pipelined buses. Appl Opt, 35:1827-1835.

[49] F. E. Kiamilev, P. Marchand, A. V. Krishnamoorthy, S. C. Esener and S. H. Lee. Performance comparison between optoelectronic and VLSI multistage interconnection networks. I Lightwave Tech, 9:1674-1692, 1991.

[50] T. J. Cloonan. Comparative study of optical and electronic interconnection technologies for large asynchronous transfer mode packet switching applications. Appl Opt, 33:1512-1523, 1994 
[51] L. J. Camp, R. Sharma, M. R. Feldman. Guided-wave and free-space optical interconnects for parallel-processing systems: a comparison. Appl Opt, 33:6168-6180, 1994.

[52] A. V. Krishnamoorthy, P. J. Marchand, F. E. Kiamilev, and S. C. Esener. Grain-size considerations for optoelectronic multistage interconnection networks. Appl Opt, 31:5480-5507, 1992.

[53] H. M. Ozaktas and J. W. Goodman. Implications of interconnection theory for optical digital computing. Appl Opt, 31:5559-5567, 1992.

[54] J. Fan, B. Catanzaro, V. H. Ozguz, C. K. Cheng, and S. H. Lee. Design considerations and algorithms for partitioning optoelectronic multichip modules. Appl Opt, 34:3116-3127, 1995.

[55] F. Kiamilev, S. C. Esener, R. Paturi, Y. Fainman, P. Mercier, C. C. Guest, and S. H. Lee. Programmable optoelectronic multiprocessors and their comparison with symbolic substitution for digital optical computing. Opt Eng, 28:396-409, 1989.

[56] D.-T. Lu, V. H. Ozguz, P. J. Marchand, A. V. Krishnamoorthy, F. Kiamilev, R. Paturi, S. H. Lee, and S. C. Esener. Design trade-offs in optoelectronic parallel processing systems using smart-SLMs. Optical and Quantum Electronics, 24:S379-S403, 1992.

[57] T. J. Drabik. Optoelectronic integrated systems based on free-space interconnects with an arbitrary degree of space-variance. Proc IEEE, 82:1595-1622, 1994.

[58] M. P. Y. Desmulliez, B. S. Wherrett, J. F. Snowdon, and J. A. B. Dines. Optical, algorithmic and electronic considerations on the desirable "smartness" of optical processing pixels. In Optical Computing, Proceedings of the International Conference, Edinburgh, 1994, Inst Phys Pub, Bristol, U.K., 1995, pp 489-492.

[59] D. R. Rolston, B. Robertson, H. S. Hinton, and D. V. Plant. Analysis of a microchannel interconnect based on the clustering of smart-pixeldevice windows. Appl Opt, 35:1220-1234, 1996.

[60] H. M. Ozaktas. Towards an Optimal Foundation Architecture for Optoelectronic Computing, Part I: Regularly Interconnected Device Planes; Part II: Physical Construction and Application Platforms. Appl Opt, to appear.

[61] H. S. Hinton, T. J. Cloonan, F. B. McCormick, A. L. Lentine, F. A. P. Tooley. Free-space digital optical systems. Proc IEEE, 82:1632$1649,1994$.

[62] H. S. Hinton. Introduction to Photonic Switching Fabrics. Plenum, New York, 1993.

[63] T. H. Szymanski and H. S. Hinton. Reconfigurable intelligent optical backplane for parallel computing and communications. Appl Opt, 35:1253-1268, 1996.

[64] K. S. Huang, C. B. Kuznia, B. K. Jenkins, and A. A. Sawchuk. Parallel architectures for digital optical cellular image processing. Proc. IEEE, 82:1711-1723, 1994.

[65] P. S. Guilfoyle. Digital optical computing architectures for compute intensive applications. In Optical Computing, Proceedings of the International Conference, Edinburgh, 1994, Inst Phys Pub, Bristol, U.K., 1995, pp 285-288.

[66] V. N. Morozov, J. A. Neff, H. Temkin, A. S. Fedor. Analysis of a three-dimensional computer optical scheme based on bidirectional free-space optical interconnects. Opt Eng, 34:523-534, 1995.

[67] L. J. Irakliotis, S. A. Feld, F. R. Beyette, Jr., P. A. Mitkas, and C. W. Wilmsen. Optoelectronic parallel processing with surface-emitting lasers and free-space interconnects. J Lightwave Tech, 13:1074-1084, 1995.

[68] A. Louri and S. Furlonge. Feasibility study of a scalable optical interconnection network for massively parallel processing systems. Appl Opt, 35:1296-1308, 1996.
[69] P. J. Marchand, A. V. Krishnamoorthy, G. I. Yayla, S. C. Esener, and U. Efron. Optically augmented 3-D computer: system technology and architecture. J Parallel and Distributed Computing, to appear.

[70] G. I. Yayla, P. J. Marchand, and S. C. Esener. Energy and speed analysis of digital electrical and free-space optical interconnections In Optical Interconnections and Parallel Processing: The Interface. Kluwer Academic Publishers, Dordrecht, The Netherlands, to appear.

[71] A. V. Krishnamoorthy and D. A. B. Miller. Firehose architectures for free-space optically-interconnected VLSI circuits. $J$ Parallel and Distributed Computing, to appear.

[72] R. Paturi, D.-T. Lu, J. E. Ford, S. C. Esener, and S. H. Lee. Parallel algorithms based on expander graphs for optical computing. Appl Opt, 30:917-927, 1991.

[73] H. M. Ozaktas and J. W. Goodman. Organization of information flow in computation for efficient utilization of high information flux communication media. Opt Commun, 89:178-182, 1992.

[74] T. J. Cloonan, G. W. Richards, A. L. Lentine, F. B. McCormick, Jr., H. S. Hinton, and S. J. Hinterlong. A complexity analysis of smart pixel switching nodes for photonic extended generalized shuffle switching networks. IEEE J Quantum Electronics, 20:619-634, 1993.

[75] D. A. B. Miller. Device requirements for digital optical processing. Digital Optical Computing, SPIE Critical Reviews, 1990.

[76] C. Fan, B. Mansoorian, D. A. Van Blerkom, M. W. Hansen, V. H. Ozguz, S. C. Esener, and G. C. Marsden. Digital free-space optical interconnections: a comparison of transmitter technologies. Appl Opt, 34:3103-3115, 1995

[77] H. M. Ozaktas, H. Urey, and A. W. Lohmann. Scaling of diffractive and refractive lenses for optical computing and interconnections. Appl Opt, 33:3782-3789, 1994.

[78] D. T. Neilson and C. P. Barrett. Performance trade-offs for conventional lenses for free-space digital optics. Appl Opt, 35:1240-1248, 1996.

[79] K. S. Urquhart, P. Marchand, Y. Fainman, and S. H. Lee. Diffractive optics applied to free-space optical interconnects. Appl Opt, 33:36703683.

[80] A. V. Krishnamoorthy and D. A. B. Miller. Scaling optoelectronicVLSI circuits into the 21 st century: a technology roadmap. IEEE $J$ Selected Topics Quantum Electronics, 2:55-76, 1996.

[81] H. M. Ozaktas. Levels of abstraction in computing systems and optical interconnection technology. In Optical Interconnections and Parallel Processing: The Interface. Kluwer Academic Publishers, Dordrecht, The Netherlands, to appear.

[82] N. Rosenberg. Exploring The Black Box: Technology, Economics. and History. Cambridge University Press, Cambridge, 1994.

We acknowledge the support of NATO under the Science for Stability Program. 